\title{
Role of Primary and Secondary Bile Acids as Feedback Inhibitors of Bile Acid Synthesis in the Rat In Vivo
}

\author{
Eduard F. Stange, Jürgen Scheibner, and Hans Ditschuneit \\ Department of Internal Medicine II, University of Ulm, 7900 Ulm, Federal Republic of Germany
}

\begin{abstract}
The effect of various primary and secondary bile acids on the rates of synthesis of all major bile acids was studied in the live rat with an extracorporal bile duct. Bile acid synthesis was determined using HPLC based on mass or by isotope dilution. Derepressed rates of bile acid synthesis (30-54 h) were inhibited by an infusion of taurocholic acid only at a supraphysiological dose of $500 \mu \mathrm{mol} / \mathrm{kg}$ per $\mathrm{h}$, but not at $300 \mu \mathrm{mol} / \mathrm{kg}$ per $h$, which approximates the initial bile acid secretion (250 $\mu \mathrm{mol} / \mathrm{kg}$ per $\mathrm{h})$. When administered together with taurocholic acid (200 $\mu \mathrm{mol} / \mathrm{kg}$ per $\mathrm{h})$ only a high dose of taurochenodeoxycholic acid (100 $\mu \mathrm{mol} / \mathrm{kg}$ per $\mathrm{h})$ decreased taurocholic but not tauromuricholic or taurochenodeoxycholic acid synthesis. The only bile acid suppressing taurocholic acid (36-71\%) and taurochenodeoxycholic acid (up to 33\%) formation at an infusion rate close to the normal portal flux was deoxy- or taurodeoxycholic acid at 15-50 $\mu \mathrm{mol} / \mathrm{kg}$ per $\mathrm{h}$. It may be concluded that deoxycholic acid and possibly other secondary bile acids are much more potent inhibitors than primary bile acids.
\end{abstract}

\section{Introduction}

The classical view that the rate of bile acid synthesis is under the control of bile acids returning to the liver via the portal vein is based on a large body of evidence. For example, both bile acid synthesis and its rate-limiting enzyme, 7a-hydroxylase, were suppressed when rats were administered a variety of bile acids through dietary, intraduodenal, or intravenous routes $(1-7)$. This mechanism explained the rapid predominance in bile of any administered bile acid (8). During partial or total interruption of the enterohepatic circulation of bile acids with cholestyramine or bile diversion bile acid synthesis increased (9-12) due to apparent derepression. These data obtained in rats were confirmed by work in primates $(13,14)$ and in humans (15-23) using both bile acid depletion and administration.

However, agreement in the field on the existence of this feedback regulation of bile acid synthesis has not been universal. First, bile acids failed to regulate their rates of synthesis

Parts of this work were presented at the Annual Meeting of the American Gastroenterological Association in Chicago, 1987, and published in abstract form.

Address correspondence to Dr. Eduard F. Stange, Department of Internal Medicine II, University of Ulm, Oberer Eselsberg, Robert Koch Strasse, 7900 Ulm, Federal Republic of Germany.

Received for publication 7 March 1988 and in revised form 4 January 1989.

J. Clin. Invest.

(c) The American Society for Clinical Investigation, Inc.

0021-9738/89/07/0173/08 \$2.00

Volume 84 , July $1989,173-180$ both in isolated $(24)$ and cultured $(25,26)$ hepatocytes. Second, several in vivo studies in the rat, hamster, and man also were unable to confirm the feedback concept uniformly (27-34). Finally, other data were similarly difficult to reconcile with the classical view, such as the stimulation of bile acid synthesis in the bile duct-ligated rat despite a 10-fold increase of hepatic bile acid concentration (35).

In an effort to gain more insight into the mechanisms of control involved in bile acid synthesis, we have recently found a distinct difference between taurocholic or cholic acid ${ }^{1}$ given as a dietary supplement as opposed to an intravenous infusion (32). It was further demonstrated that colectomy stimulated basal bile acid synthesis in the rat severalfold and partly prevented the suppressive action of dietary cholate. Taken together these findings pointed to an important role of colon-derived secondary bile acids as feedback regulators. The present study, therefore, systematically defined the role of primary and secondary bile acids in regulating hepatic synthesis of individual bile acids in the rat with an extracorporal bile duct.

\section{Methods}

Animals and surgical procedures. Male Wistar derived rats (ChbbTHOM; Thomae, Biberach, FRG) weighing 250-300 g were given a pelleted standard diet (Altromin 1314; Altromin, Lage, FRG) ad lib. After 1 wk under light cycling conditions an extracorporal bile duct was established as described by Weis and Barth (36).

Experimental setup and infusates. After another week with intact enterohepatic circulation the animals were restrained in a Bollmann cage. The external loop was cut and connected to a PE 86 tubing on both ends for duodenal infusion and bile drainage via the distal and proximal bile duct, respectively. Bile was collected at 3-h intervals using a fraction collector.

While in the restraining cage the animals were administered food and water ad lib. The average food consumption was $15 \mathrm{~g} / \mathrm{d}$ and did not differ between groups. In addition, they were infused with a standard solution including $33.8 \mathrm{mM}$ bicarbonate (32). The various bile acids (Sigma Chemical GmbH, Munich, FRG) were added to the complete infusate at concentrations chosen to achieve the required dose at an infusion rate of $2 \mathrm{ml} / \mathrm{h}$ using a precision pump (Unita Ib; B. Braun, Melsungen, FRG).

Finally, labeled bile acids were included as specified to allow for the calculation of recoveries and synthesis rates of the infused bile acids. $\left[{ }^{14} \mathrm{C}\right]$ Taurocholic acid $(46.7 \mathrm{mCi} / \mathrm{mmol}),\left[{ }^{3} \mathrm{H}\right]$ taurocholic acid $(2.7 \mathrm{Ci} /$ $\mathrm{mmol})$, and $\left[{ }^{14} \mathrm{C}\right] \mathrm{cholic}$ acid $(52 \mathrm{mCi} / \mathrm{mmol})$ were obtained from New England Nuclear (Dreieichenhain, FRG) and $\left[{ }^{14} \mathrm{C}\right]$ deoxycholic acid $(58 \mathrm{mCi} / \mathrm{mmol})$ was purchased from Amersham Corp. (Braunschweig, FRG). $\left[{ }^{14} \mathrm{C}\right]$ Taurochenodeoxycholic acid was prepared by injecting a

1. The following common names have been substituted for the systematic names in this paper: cholesterol, 5-cholesten-3 $\beta$-ol; muricholic acid, 3,6,7-trihydroxy- $5 \beta$-cholan-24-oic acid; cholic acid, 3a,7a,12atrihydroxy-5 $\beta$-cholan-24-oic acid; chenodeoxycholic acid, 3a,7a-dihydroxy-5 $\beta$-cholan-24-oic acid; deoxycholic acid, 3a, 12a-dihydroxy- $5 \beta$ cholan-24-oic acid. 
rat with $\left[{ }^{14} \mathrm{C}\right]$ chenodeoxycholic acid $(50 \mathrm{mCi} / \mathrm{mmol}$; Amersham Corp.) intravenously and subsequently isolating the labeled taurine conjugate of chenodeoxycholic acid by HPLC as described below.

A micellar solution of $46.7 \mathrm{mM}$ taurocholic acid, $0.3 \mathrm{mM}$ cholesterol, and $4.9 \mathrm{mM}$ dipalmitoyl lecithin (all from Sigma Chemical $\mathrm{GmbH}$ ) was prepared as described by Stone et al. (37). For a separate set of experiments pooled rat bile from normal animals with a bile fistula for up to $18 \mathrm{~h}$ was lyophilized and the residue dissolved in an infusate volume adequate for the required bile acid dose. The total bile acid concentration was $48.3 \mathrm{mM}$ with tauromuricholic acid accounting for $11.3 \%$ of bile acid mass, taurocholic acid $85.9 \%$, taurochenodeoxycholic acid $1.5 \%$, taurodeoxycholic acid $0.5 \%$, and cholic acid $0.8 \%$. The concentrations of cholesterol and phospholipids were 0.36 and $5.7 \mathrm{mM}$, respectively. In the various experiments listed in Table I the recoveries in bile of the infused labeled bile acids varied between 86.2 and $104 \%$.

Two different types of experiments were performed. In the first set bile acids were infused for $54 \mathrm{~h}$ and bile acid synthesis was quantitated during the derepressed phase between $30-54 \mathrm{~h}$ under continuous infusion directly using HPLC (mass), or by isotope dilution using HPLC with on-line liquid scintillation counting. In the second set of experiments bile acids were infused for $54 \mathrm{~h}$, followed by a tracer dose during the final 54-78 $\mathrm{h}$ of the experiment, allowing direct estimation of synthesis from the secreted bile acid mass after depletion of the infused bile acid pool after $60 \mathrm{~h}$. At the end of the experiments the animals were bled and glutamate-pyruvate transaminase (SGPT), ${ }^{2}$ glutamate dehydrogenase (GLDH), and alkaline phosphatase activity were determined using Merckotest kits (E. Merck, Darmstadt, FRG).

Analytical methods. Bile acids were extracted from $0.5 \mathrm{ml}$ of fresh bile according to Tietz et al. (38). The various bile acids were quantitated by HPLC as described previously (32) using a system with a differential refractometer (Waters Associates, Milford, MA) and a Lichrosorb RP 18 column (E. Merck). The radioactivity of the eluting bile acid peaks was measured directly using an on-line liquid scintillation counter (Ramona 4 LS; Raytest, Pforzheim, FRG). Biliary lipids were assayed as detailed (32).

Calculations. The synthesis rates of those bile acids that were not administered or metabolites of infused bile acids were derived directly from their rates of mass secretion after pool depletion. In contrast, the synthesis rates of the bile acids infused were calculated using the Wilson formula (28) based on the difference in specific activity in bile and infusate:

Bile acid synthesis $(\mu \mathrm{mol} / \mathrm{h})$

$=\frac{\mathrm{dpm} / \mathrm{h} \text { (bile) }}{\mathrm{dpm} / \mu \mathrm{mol} \text { (bile) }}-\frac{\mathrm{dpm} / \mathrm{h} \text { (bile) }}{\mathrm{dpm} / \mu \mathrm{mol} \text { (infusate) }}$.

In previous work (32) it was established that this measurement is valid provided the specific activity difference assayed by HPLC and scintillation counting exceeds $3 \%$. In the case of partial conversion of certain precursor bile acids to other bile acid metabolites, e.g., chenodeoxycholic acid to muricholic acid or deoxycholic acid to cholic acid, this contribution was similarly assessed by measuring the radioactivity of the respective precursor label in the product. Since the specific activity of the infused precursor was known, its metabolism could be quantitated and subtracted from the total amount of product secreted to yield the rate of de novo synthesis of the product bile acid. The statistical analysis was performed using the $t$ test for unpaired samples.

\section{Results}

Bile acid secretion and synthesis under control conditions. To assess the in vivo rates of bile acid secretion, bile was collected

2. Abbreviations used in this paper: GLDH, glutamate dehydrogenase; SGPT, glutamate pyruvate transaminase. during the initial $30 \mathrm{~min}$ after interrupting the extracorporal bile duct. With 134 and $91 \mu \mathrm{mol} / \mathrm{kg}$ per $\mathrm{h}$, tauromuricholic acid and taurocholic acid were the two major bile acids secreted (Fig. 1). Minor bile acids were taurochenodeoxycholic acid $(10.3 \pm 1.8$, means \pm SEM), taurohyodeoxycholic acid (8.3 \pm 2.2$)$, taurodeoxycholic acid (5.8 \pm 1.3$)$, and tauroursodeoxycholic acid $(0.8 \pm 0.3)$. Thus, total bile acid secretion amounted to $250 \mu \mathrm{mol} / \mathrm{kg}$ per $\mathrm{h}$ in these animals. After the nadir between 6 and $9 \mathrm{~h}$ the derepression of synthesis was proportionally much greater for taurocholic than for taurochenodeoxycholic acid, and even less for tauromuricholic acid. Conspicuously, all three major primary bile acids exhibited a distinct diurnal rhythm, but the time of peak activity was not synchronous but dissociated by 3-6 h.

Bile acid secretion and synthesis during the infusion of taurocholic acid or cholic acid with and without cholesterol. During the infusion of $\left[{ }^{14} \mathrm{C}\right]$ taurocholic acid no radioactivity was detectable in tauromuricholic or taurochenodeoxycholic acid. Thus, after pool depletion the secretion rates of these bile acids were identical with their rate of de novo synthesis even in the presence of a taurocholic acid infusion.

Surprisingly, neither taurocholic acid nor cholic acid effectively suppressed the derepressed tauromuricholic or taurochenodeoxycholic acid synthesis even at a dose close to 300 $\mu \mathrm{mol} / \mathrm{kg}$ per $\mathrm{h}$ (Fig. 2). It should be noted that this dose is higher than the normal rate of total bile acid secretion in these rats and also exceeds control taurocholic acid secretion threefold. Even a dose of $503 \mu \mathrm{mol} / \mathrm{kg}$ per $\mathrm{h}$ administered for $54 \mathrm{~h}$ did not affect tauromuricholic acid synthesis at $60-63 \mathrm{~h}$ after washout of the infused bile acid, although taurochenodeoxycholic acid formation was decreased by $35 \%$ (Fig. 3).

Similarly, taurocholic acid synthesis was not altered during the infusion of $302 \mu \mathrm{mol} / \mathrm{kg}$ per h of taurocholic acid (Figs. 2 and 3 ) and the slight decrease (28\%) during the infusion of a comparable dose of cholic acid was not statistically significant (Fig. 2). However, at the high dose of $503 \mu \mathrm{mol} / \mathrm{kg}$ per $\mathrm{h}$ of taurocholic acid given for $54 \mathrm{~h}$ (Fig. 3), taurocholic acid nadir secretion (i.e., synthesis) was inhibited by $33 \%$. Taurodeoxycholic acid appeared in bile at a rate of $2 \mu \mathrm{mol} / \mathrm{kg}$ per $\mathrm{h}$ during this infusion (Table I). Both bile flow and cholesterol as well as phospholipid secretion were stimulated during the bile acid infusions, particularly during the infusion of cholate (Table I).

In an attempt to rule out specific effects of whole bile as opposed to bile acids alone, a micellar solution of taurocholic acid (308 $\mu \mathrm{mol} / \mathrm{kg}$ per h), phospholipids (32 $\mu \mathrm{mol} / \mathrm{kg}$ per h) and cholesterol $(2.1 \mu \mathrm{mol} / \mathrm{kg}$ per $\mathrm{h})$ was infused. Under these experimental conditions both tauromuricholic and taurochenodeoxycholic acid synthesis were slightly inhibited (by 23 and $26 \%$, respectively), but taurocholic acid synthesis again was unchanged (Fig. 2). Comparable results were obtained with lyophilized rat bile adjusted to a total bile acid infusion dose of $321 \mu \mathrm{mol} / \mathrm{kg}$ per h (data not shown).

Bile acid secretion and synthesis during the infusion of taurocholic plus taurochenodeoxycholic acid. In another series of experiments the effect of taurochenodeoxycholic acid on rates of bile acid synthesis was determined in the presence of taurocholic acid to reduce the potential toxicity of the former bile acid. At the lower dose of $53 \mu \mathrm{mol} / \mathrm{kg}$ per $\mathrm{h}$ of taurochenodeoxycholic acid plus 263 of taurocholate, the rate of tauromuricholic acid formation was even stimulated despite correction for the flux of taurochenodeoxycholic acid into tauromuricholic acid (Fig. 4), but the other bile acids were 
Table I. Bile Flow and Biliary Lipid Secretion during Bile Acid Infusions

\begin{tabular}{|c|c|c|c|c|c|c|}
\hline \multirow[b]{2}{*}{ Bile acid infused } & \multirow[b]{2}{*}{ Dose } & \multirow[b]{2}{*}{$n$} & \multirow[b]{2}{*}{ Bile flow } & \multicolumn{3}{|c|}{ Secretion rates } \\
\hline & & & & TDC & Chol. & P.L. \\
\hline & umol/kg per $h$ & & $m l / h$ & & umol/kg per $h$ & \\
\hline TC (Control) & 1 & 21 & $0.7 \pm 0.02$ & n.d. & $0.7 \pm 0.03$ & $10.7 \pm 0.4$ \\
\hline TC & 302 & 13 & $1.6 \pm 0.1^{*}$ & n.d. & $2.0 \pm 0.2^{*}$ & $32.4 \pm 1.3^{*}$ \\
\hline TC & 503 & 's & $2.3 \pm 0.1^{*}$ & $2.0 \pm 0.8$ & $1.3 \pm 0.1^{*}$ & $40.7 \pm 1.8^{*}$ \\
\hline $\mathrm{C}$ & 310 & 6 & $1.6 \pm 0.1^{*}$ & n.d. & $2.3 \pm 0.1^{*}$ & $42.6 \pm 1.1^{*}$ \\
\hline $\mathrm{TC}+\mathrm{Chol}+$ P.L. & $308+2+32$ & 11 & $1.6 \pm 0.1^{*}$ & n.d. & $2.0 \pm 0.2^{*}$ & $27.8 \pm 1.8^{*}$ \\
\hline Whole bile & 321 & 10 & $1.8 \pm 0.05^{*}$ & n.d. & $1.9 \pm 0.1^{*}$ & $40.8 \pm 0.6^{*}$ \\
\hline \multirow[t]{2}{*}{$\mathrm{TC}+\mathrm{TCDC}$} & $263+53$ & 7 & $1.7 \pm 0.1^{*}$ & n.d. & $1.8 \pm 0.1^{*}$ & $35.7 \pm 2.6^{*}$ \\
\hline & $205+102$ & 9 & $1.3 \pm 0.1^{*}$ & n.d. & $1.9 \pm 0.1^{*}$ & $36.6 \pm 1.3^{*}$ \\
\hline $\mathrm{TC}+\mathrm{DC}$ & $285+15$ & 5 & $1.7 \pm 0.03^{*}$ & $6.7 \pm 0.9$ & $1.6 \pm 0.03^{*}$ & $26.9 \pm 0.7^{*}$ \\
\hline $\mathrm{TC}+\mathrm{DC}$ & $259+24$ & 9 & $1.3 \pm 0.04^{*}$ & $10.1 \pm 0.6$ & $2.1 \pm 0.2^{*}$ & $29.2 \pm 1.2^{*}$ \\
\hline $\mathrm{TC}+\mathrm{DC}$ & $246+49$ & 11 & $1.2 \pm 0.04^{*}$ & $26.8 \pm 1.2$ & $2.2 \pm 0.2^{*}$ & $30.7 \pm 1.4^{*}$ \\
\hline $\mathrm{TC}+\mathrm{TDC}$ & $253+51$ & 6 & $1.4 \pm 0.04^{*}$ & $32.6 \pm 1.8$ & $2.0 \pm 0.2^{*}$ & $34.4 \pm 1.6^{*}$ \\
\hline DC & 51 & 8 & $0.9 \pm 0.05^{\ddagger}$ & $28.6 \pm 1.5$ & $0.7 \pm 0.1$ & $17.8 \pm 2.3^{*}$ \\
\hline
\end{tabular}

Groups of animals with an extracorporal bile duct for $1 \mathrm{wk}$ before the experiment were infused with taurocholic acid (TC), cholic acid (C), taurocholic acid + cholesterol + phospholipid (TC + Chol + P.L.), whole bile, taurochenodeoxycholic acid (TCDC), deoxycholic acid (DC), taurodeoxycholic acid (TDC), or combinations thereof at the doses indicated via the distal bile duct. Bile was collected from the proximal bile duct during the period between 30 and $54 \mathrm{~h}$ after interrupting the enterohepatic circulation. Each value represents the mean \pm 1 SEM obtained in $n$ animals. $\quad{ }^{*} P<0.01 ;^{\ddagger} P<0.05$ compared with controls (TC $1 \mu \mathrm{mol} / \mathrm{kg}$ per h); n.d., not detectable.

unaffected. The higher dose of 102 plus $205 \mu \mathrm{mol} / \mathrm{kg}$ per h of taurochenodeoxycholic and taurocholic acid, respectively, had a similar effect, except that in addition at this level taurocholic acid production was decreased by $55 \%$. These findings were confirmed in the 78-h experiment (Fig. 3).

Bile acid secretion and synthesis during the infusion of deoxycholic or taurodeoxycholic acid with and without taurocholic acid. Deoxycholic acid infused at rates of $49-51 \mu \mathrm{mol} /$ $\mathrm{kg}$ per $\mathrm{h}$ slightly stimulated tauromuricholic acid synthesis be-

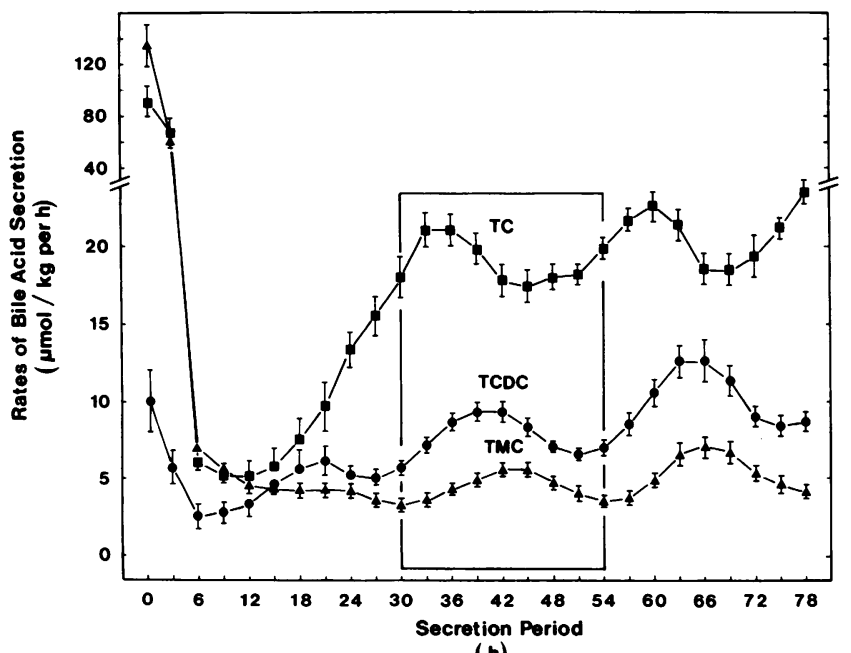

(h)

Figure 1. Rates of taurocholic (TC), taurochenodeoxycholic $(T C D C)$, and tauromuricholic acid $(T M C)$ secretion after interruption of the enterohepatic circulation via the extracorporal bile duct. The initial rates are given for the $0-0.5$-h period; after $3 \mathrm{~h}$ the bile was collected during 3-h intervals. Values represent the means \pm 1 SEM of 11 animals. The frame denotes the period during which bile acid synthesis was measured in Figs. 2, 4, and 5.

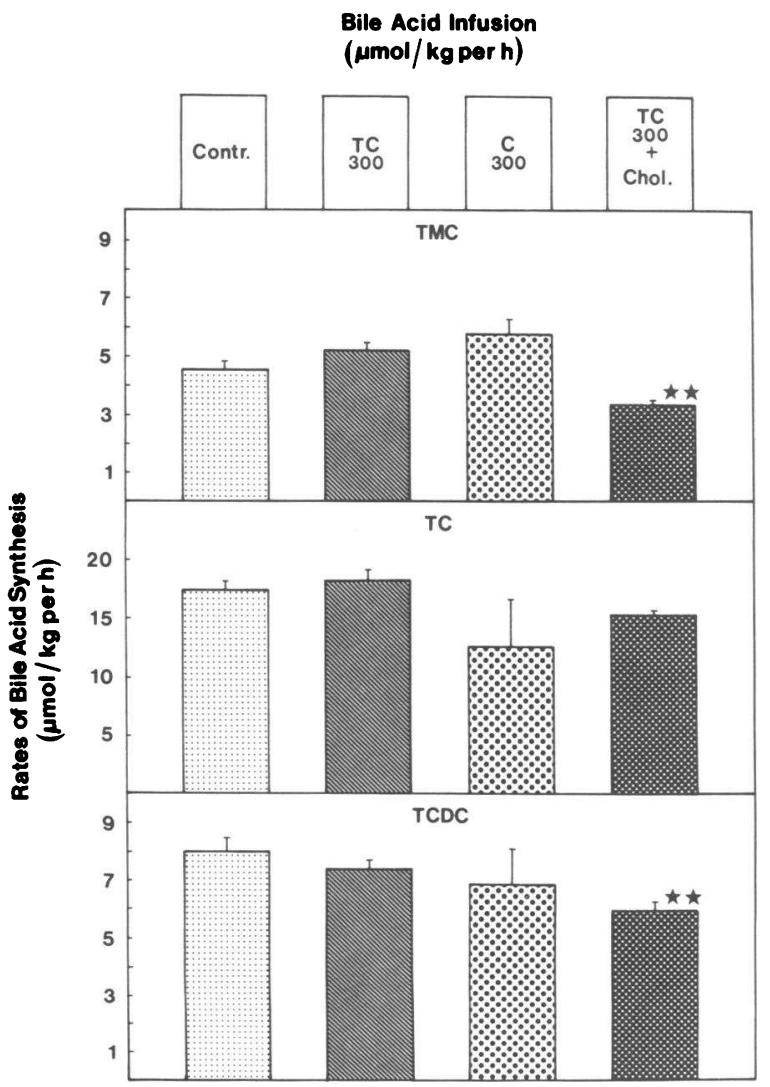

Figure 2. Rates of tauromuricholic $(T M C)$, taurocholic $(T C)$, and taurochenodeoxycholic acid $(T C D C)$ synthesis at 30-54 $\mathrm{h}$ during the infusions of taurocholic acid $(T C)$, cholic acid, $(C)$ or taurocholic acid plus cholesterol plus phospholipids $(T C+C h o l)$. The precise doses normalized to animal weights and the numbers of animals per group are detailed in Table I. Values represent the means \pm 1 SEM. ${ }_{* *}^{*} P<0.01$ vs. controls. 


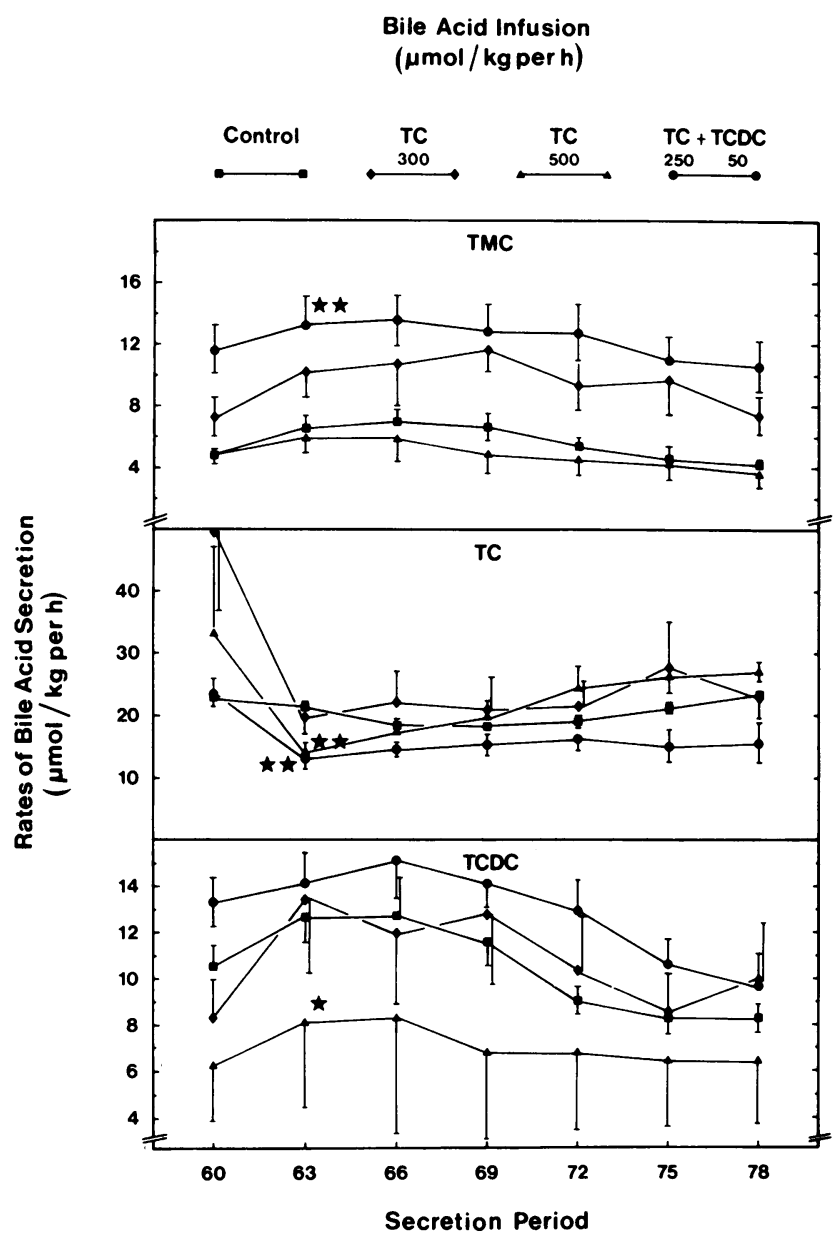

(h)

Figure 3. Rates of tauromuricholic (TMC), taurocholic (TC), and taurochenodeoxycholic acid $(T C D C)$ secretion between 60 and $78 \mathrm{~h}$ after interruption of bile acid infusions at $54 \mathrm{~h}$. The precise doses given were $1 \mu \mathrm{mol} / \mathrm{kg}$ per h of taurocholic acid (Control, $n=11$ ), 302 of taurocholic acid (TC, $n=13), 503$ of taurocholic acid (TC, $n$ $=5$ ), and 263 of taurocholic acid plus 53 of taurochenodeoxycholic acid $(T C+T C D C, n=7)$. Values represent the means \pm 1 SEM. ${ }^{*} P$ $<0.05 ;{ }^{* *} P<0.01$ vs. controls.

tween 30 and $54 \mathrm{~h}$, but suppressed taurocholic acid formation by $71 \%$, more than any other bile acid at any dose. In the presence of additional taurocholic acid, its effect on taurocholic acid synthesis was even more pronounced and also extended to taurochenodeoxycholic acid $(-33 \%)$ (Fig. 5).

During the phase after cessation of the infusion, a combination of taurocholic acid and deoxycholic acid at lower doses (254 plus $24 \mu \mathrm{mol} / \mathrm{kg}$ per h or 285 plus $15 \mu \mathrm{mol} / \mathrm{kg}$ per h, respectively) also inhibited nadir secretion (i.e., synthesis) of taurocholic acid by 41 and $36 \%$, respectively (Fig. 6). By contrast, both tauromuricholic and taurochenodeoxycholic acid synthesis remained unaffected at these relatively low doses. In comparison, after administration of taurocholic acid plus taurodeoxycholic acid the decrease in taurocholic acid synthesis was similar, but again the changes in tauromuricholic and taurochenodeoxycholic acid synthesis were not statistically significant.

As judged from the amount of $\left[{ }^{14} \mathrm{C}\right]$ deoxycholic acid label appearing in taurocholic acid, a significant proportion $(40-56 \%)$ of deoxycholic acid was rehydroxylated and conju-

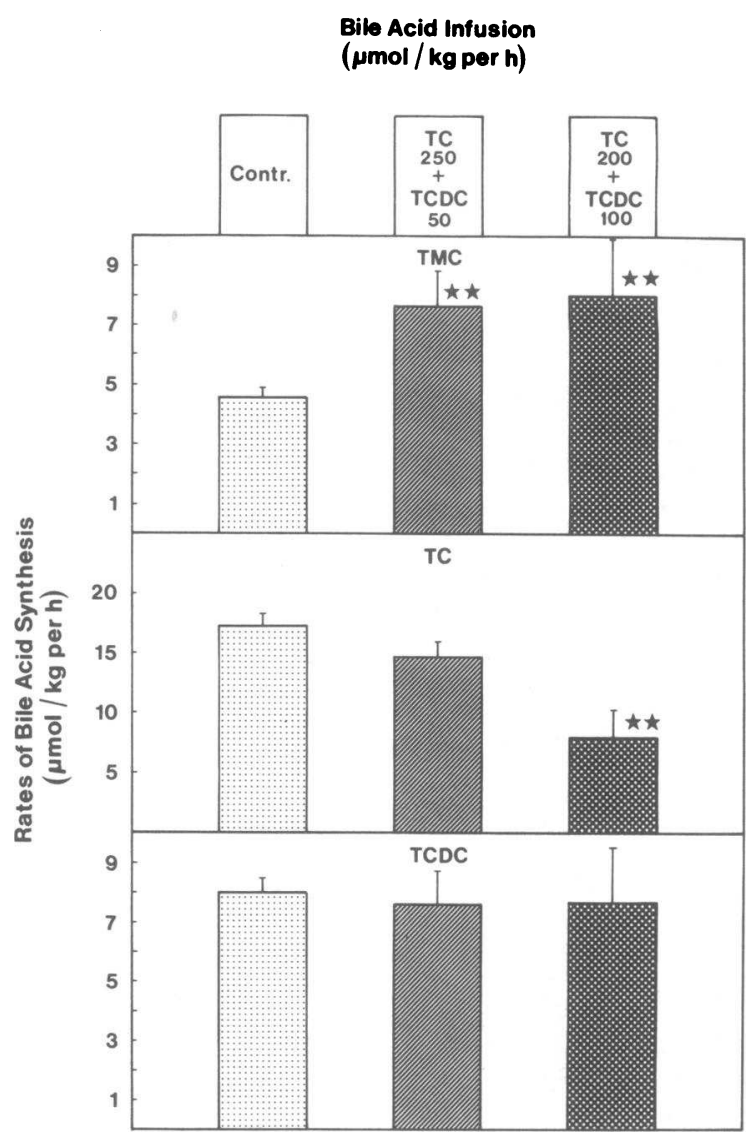

Figure 4. Rates of tauromuricholic (TMC), taurocholic (TC), and taurochenodeoxycholic acid $(T C D C)$ synthesis at 30-54 $\mathrm{h}$ during the infusions of taurocholic acid plus taurochenodeoxycholic acid (TC $+T C D C)$ as detailed in Table $I$. Values represent the means \pm 1 SEM. ${ }^{* *} P<0.01$ vs. controls.

gated during its liver passage to taurocholic acid. The presence or absence of $246-259 \mu \mathrm{mol} / \mathrm{kg}$ per $\mathrm{h}$ of taurocholic acid did not affect this process, but the proportion was highest $(55-56 \%)$ at the lowest doses of deoxycholic acid. Thus, the amounts of deoxycholic acid secreted in bile were only a fraction of the amount infused, despite excellent $(102 \%)$ recovery of infused deoxycholic acid derived ${ }^{14} \mathrm{C}$-label in bile (Table I).

Finally, it is important to note that none of the various bile acids administered adversely affected SGPT, GLDH, or alkaline phosphatase levels (Table II). On the other hand, although the various bile acids appeared to differ with respect to their choleretic effect, none clearly diminished bile flow as an indication of a cholestatic effect.

\section{Discussion}

Several methodological aspects of the present work deserve emphasis. Most importantly, the current rat model with an extracorporal bile duct as detailed by Weis and Barth (36) avoids problems with surgical stress immediately preceding the experiment. With extracorporal bile duct techniques $(32,36$, 39) the rates of hepatic bile acid secretion were much higher (250-400 $\mu \mathrm{mol} / \mathrm{kg}$ per h) and probably more in the physiological range than reported previously $(11,28)$. Therefore, it was necessary to infuse relatively large amounts of bile acids to 


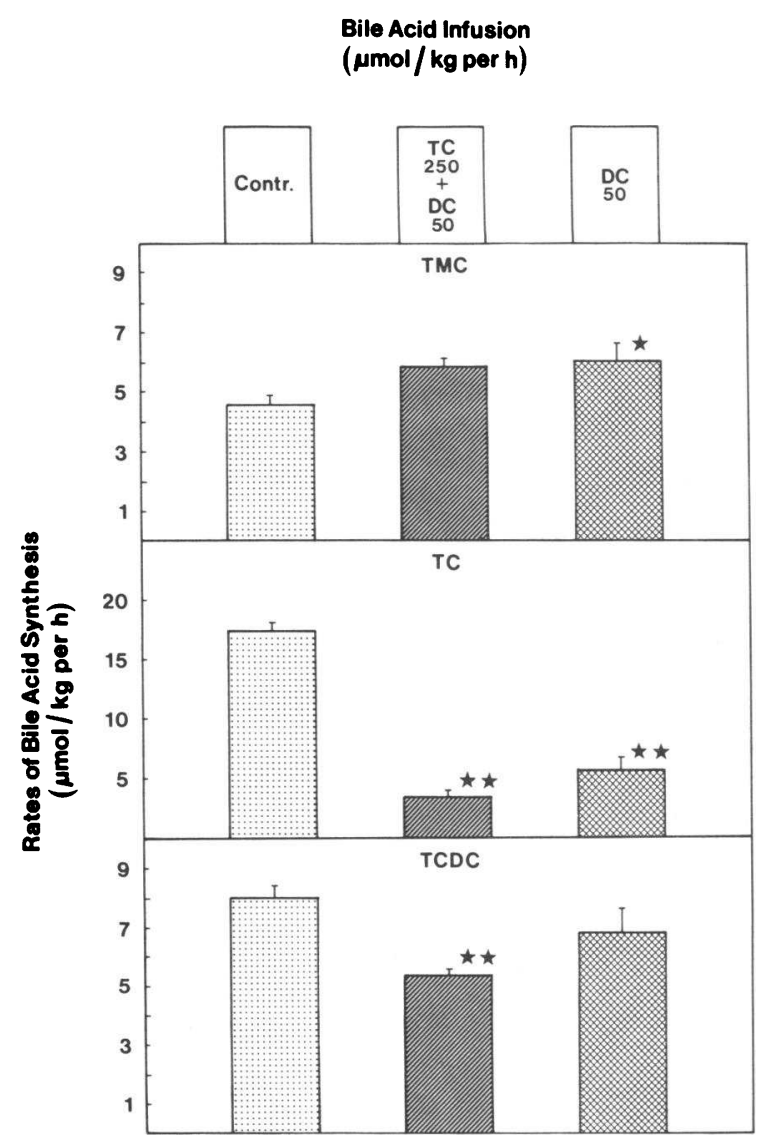

Figure 5. Rates of tauromuricholic (TMC), taurocholic (TC), and taurochenodeoxycholic acid (TCDC) synthesis at 30-54 h during the infusions of taurocholic plus deoxycholic acid $(T C+D C)$ and deoxycholic acid $(D C)$ alone as detailed in Table I. Values represent the means \pm 1SEM. ${ }^{*} P<0.05 ;{ }^{* *} P<0.01$ vs. controls.

provide a physiological rate of transhepatic flux, since principally at least maintenance of this flux should be necessary to prevent the derepression of bile acid synthesis.

Both the extracorporal bile duct technique and a complex infusion mixture including bicarbonate to avoid metabolic acidosis $(30,32)$ may have contributed to the virtual lack of bile acid toxicity in the present studies. It should also be emphasized that in this study potentially toxic hydrophobic bile acids like taurochenodeoxycholic or deoxycholic acid $(40,41)$ were antagonized by a concomitant supply of the more hydrophilic and less toxic (40) taurocholic acid. The lack of hepatic toxicity was documented by near normal SGPT, GLDH, and alkaline phosphatase activity at the end of the experiment. None of the bile acid infusions reduced bile flow or the secretion of biliary lipids as compared with controls. In addition, the effects on bile acid synthesis were specific, since taurocholic acid formation was more sensitive to feedback inhibition by the dihydroxy bile salts than taurochenodeoxycholic acid, and tauromuricholic acid was completely spared. Such a differential response is not consistent with overall hepatic toxicity of these agents.

Taurocholic acid, as reported for the acute bile fistula rat (32), was formed at normal rates even in the presence of a taurocholic acid infusion rate up to $300 \mu \mathrm{mol} / \mathrm{kg}$ per $\mathrm{h}$ (i.e., more than threefold higher than the taurocholic acid secretion

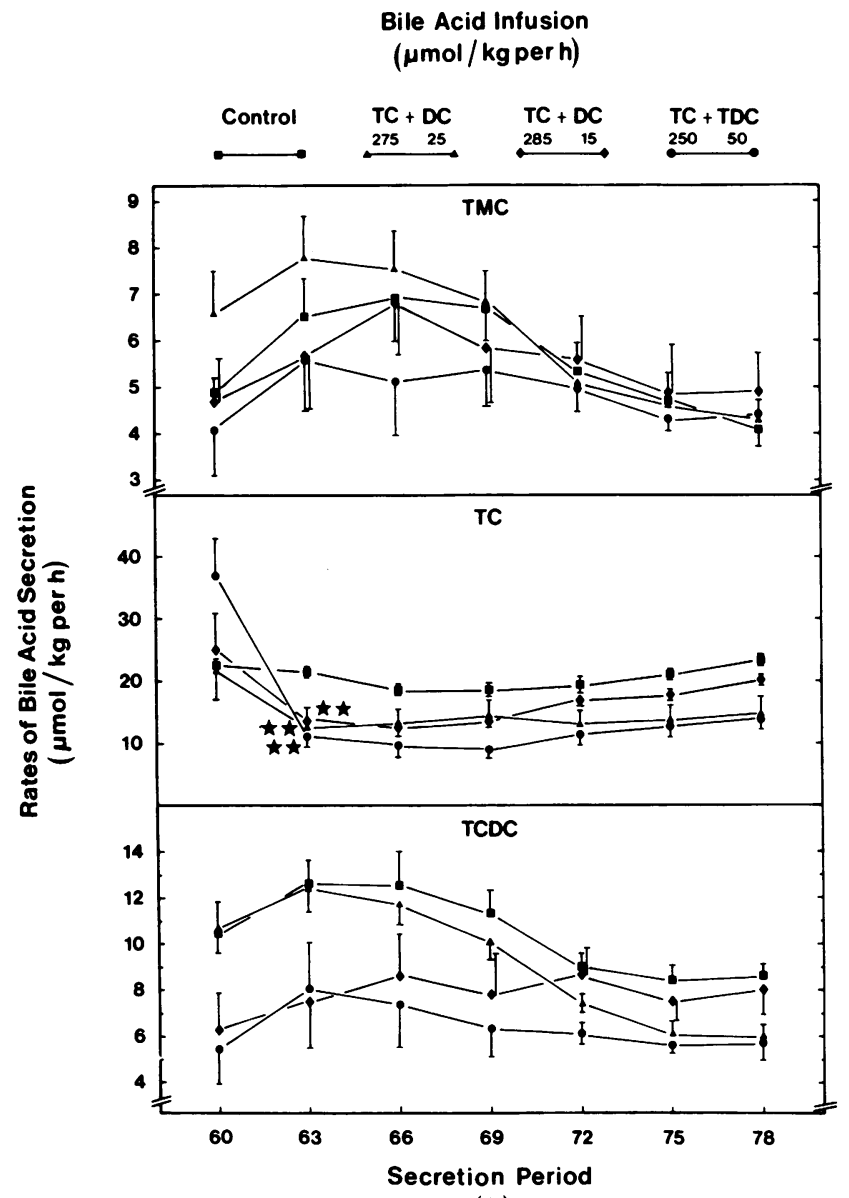

(h)

Figure 6. Rates of tauromuricholic (TMC), taurocholic (TC), and taurochenodeoxycholic acid $(T C D C)$ secretion between 60 and $78 \mathrm{~h}$ after interruption of bile acid infusions at $54 \mathrm{~h}$. The precise doses given were $1 \mu \mathrm{mol} / \mathrm{kg}$ per $\mathrm{h}$ of taurocholic acid (Control, $n=11$ ), 285 of taurocholic acid plus 15 of deoxycholic acid $(T C+D C, n$ $=5), 259$ of taurocholic plus 24 of deoxycholic acid $(T C+D C, n$ $=9)$, and 253 of taurocholic plus 51 of taurodeoxycholic acid $(T C+$ $T D C, n=6)$. Values represent the means \pm 1 SEM. ${ }^{* *} P<0.01$ vs. controls.

and equivalent to total bile acid secretion in the extracorporal bile duct animals). However, at a very high dose of $\sim 500$ $\mu \mathrm{mol} / \mathrm{kg}$ per $\mathrm{h}$, which was accompanied by the appearance of

Table II. SGPT, GLDH, and Alkaline Phosphatase Activity in Serum after Bile Acid Infusions

\begin{tabular}{lcclc}
\hline \multicolumn{1}{c}{ Group } & Dose & SGPT & \multicolumn{1}{c}{ GLDH } & Alkaline phosphatase \\
\multicolumn{4}{c}{$\mu$ mol/kg per $h$} \\
Normals & - & $22 \pm 0.8$ & $1.0 \pm 0.1$ & $241 \pm 15$ \\
TC & 302 & $27 \pm 1.6$ & $0.8 \pm 0.07$ & $199 \pm 6$ \\
TC + TCDC & $205 \pm 102$ & $24 \pm 1.5$ & $1.4 \pm 0.09^{*}$ & $207 \pm 33$ \\
TC + DC & $246 \pm 49$ & $19 \pm 1.9$ & $1.3 \pm 0.3$ & $238 \pm 35$ \\
DC & 51 & $23 \pm 0.8$ & $1.1 \pm 0.3$ & $128 \pm 11$
\end{tabular}

Serum enzyme activity was measured in a group of normal animals $(n=19)$ and in the groups detailed in Table I after the bile acid infusions. Each value represents the mean \pm 1 SEM.

${ }^{*} P<0.01$ compared with normals. 
deoxycholate in bile, taurocholic acid synthesis was indeed suppressed. Thus, taurocholic acid did not act as a strong inhibitor of its own synthesis.

The unconjugated cholate was somewhat more effective, but the slight suppression achieved was not statistically significant. Similar to taurocholic and cholic acid, the inhibitory doses of taurochenodeoxycholic acid (100 $\mu \mathrm{mol} / \mathrm{kg}$ per $\mathrm{h})$ exceeded the rates of normal biliary secretion 10-fold, suggesting that this bile acid also is not a physiological inhibitor.

In contrast, both deoxycholate and its taurine conjugate were found to be very effective inhibitors of taurocholic acid formation at much lower doses between 15 and $50 \mu \mathrm{mol} / \mathrm{kg}$ per $\mathrm{h}$ both in the absence or presence of additional taurocholic acid. Several points argue in favor of a physiological role of this secondary bile acid. First, the minimal effective dose of deoxycholic acid used in this study of $15 \mu \mathrm{mol} / \mathrm{kg}$ per $\mathrm{h}$ was rehydroxylated efficiently in the liver, leaving only $7 \mu \mathrm{mol} / \mathrm{kg}$ per $\mathrm{h}$ for secretion in bile. This is quite close to the normal rate of deoxycholic acid secretion of $6 \mu \mathrm{mol} / \mathrm{kg}$ per $\mathrm{h}$ in these animals, which is almost certainly an underestimate of the physiological flux to the liver. This flux is difficult to determine since data on bile acid concentrations in rat portal blood are scanty $(24,42)$. We have therefore determined deoxycholic acid levels in postprandial rat portal serum by gas liquid chromatography and found a mean of $21.7 \mu \mathrm{M}(n=8)$. If this concentration is related to previous measurements of portal blood flow in the rat (43) a deoxycholic acid flux to the rat liver of $23.4 \mu \mathrm{mol} / \mathrm{kg}$ per $h$ may be calculated. This value is comparable to the minimal effective doses of 15-25 $\mu \mathrm{mol} / \mathrm{kg}$ per $\mathrm{h}$ of deoxycholic acid used in the present study.

Second, the stimulation of bile acid synthesis after elimination of secondary bile acids by colectomy and the reduction of the effect of cholic acid feeding in these animals is also consistent with an important regulatory role of these secondary bile acids in the live rat (32). Moreover, the induction of bile acid formation during bile duct ligation despite an elevation of bile acid concentrations in the liver (35) may also be explained, since under these conditions deoxycholic acid formation in the colon is prevented. The findings of the present study are also compatible with the absence of feedback regulation by primary bile acids in isolated or cultured hepatocytes (24-26), where deoxycholic acid is too toxic to allow the direct experiment. Finally, it is striking that the inhibition of synthesis by deoxycholate of different bile acids (taurocholate $>$ taurochenodeoxycholate $>$ tauromuricholate) follows the same order as the degree of derepression (Fig. 1), also suggesting a physiological role of this secondary bile acid.

It is quite possible that the disagreement in the literature on the question of a feedback regulation during intraduodenal taurocholate infusion is due to variable formation of secondary deoxycholate $(1,5,27-32)$. The lack of effect of taurocholate at a dose of 240-300 $\mu \mathrm{mol} / \mathrm{kg}$ per $\mathrm{h}$ is in agreement with recent work in the field (29-32), but doses ranging from 360 (31) to 500 in the present study clearly were suppressive. However, it is possible that this effect was also mediated by deoxycholate that appeared in bile.

Whether the proposed regulation by deoxycholic acid and possibly other secondary rather than primary bile acids holds true in other species is still unresolved, but recent findings in the rabbit (44) are compatible with this concept. In the hamster deoxycholic acid appears to be extremely toxic (45), whereas in the baboon deoxycholic acid was the single most potent feedback inhibitor of bile acid synthesis (14). In man, most $(16,17,19)$ but not all $(34)$ of the available evidence supports a role for deoxycholic acid as feedback inhibitor. It has also been suggested that this bile acid is instrumental not only in regulating bile acid pool sizes but also in affecting biliary cholesterol saturation (46-49). This may well have a bearing on cholesterol gallstone disease, since in some studies there was a relationship between deoxycholic acid and cholesterol in bile (50). On the other hand, at least a subgroup of gallstone patients has low rates of bile acid synthesis (51) and low pool sizes (52). Moreover, clinical situations with an increased bile acid loss through dysfunction or resection of the terminal ileum are accompanied by a compensatory increase in bile acid synthesis (53) and are often characterized by a low deoxycholate pool size (54).

In conclusion, deoxycholic acid appears to be a major determinant of taurocholic and taurochenodeoxycholic acid synthesis in the rat and possibly in other species. However, the modulation of the synthesis of the various primary bile acids in the rat differs, suggesting that their regulation is not coordinated by the same mechanisms of control.

\section{Acknowledgments}

The expert technical assistance of Mrs. E. Preiss and Mrs. H. Maucher is gratefully acknowledged.

This study was supported by the Deutsche Forschungsgemeinschaft (Sta 172/3-3).

\section{References}

1. Shefer, S., F. W. Cheng, S. Hauser, A. K. Batta, and G. Salen. 1981. Regulation of bile acid synthesis: measurement of cholesterol 7alpha-hydroxylase in rat liver microsomal preparations in the absence of endogenous cholesterol. J. Lipid Res. 22:532-536.

2. Danielsson, H. 1973. Influence of dietary bile acids on formation of bile acids in rat. Steroids. 22:667-676.

3. Danielsson, H., and G. Johansson. 1974. Effects of long term feeding of chenodeoxycholic acid on biosynthesis and metabolism of bile acids in the rat. Gastroenterology. 67:126-134.

4. Shefer, S., S. Hauser, I. Bekersky, and E. H. Mosbach. 1969. Feedback regulation of bile acid biosynthesis in the rat. J. Lipid Res. 10:646-655.

5. Shefer, S., S. Hauser, I. Bekersky, and E. H. Mosbach. 1970. Biochemical site of regulation of bile acid biosynthesis in the rat. $J$. Lipid Res. 11:404-411.

6. Raicht, R. F., B. I. Cohen, A. Sarwal, and M. Takahashi. 1978. Ursodeoxycholic acid: effects on sterol metabolism in rats. Biochim. Biophys. Acta. 531:1-8.

7. Pries, J. M., A. Gustafson, D. Wiegand, and W. C. Duane. 1983. Taurocholate is more potent than cholate in suppression of bile salt synthesis in the rat. J. Lipid Res. 24:141-146.

8. Spady, D. K., E. F. Stange, L. E. Bilhartz, and J. M. Dietschy. 1986. Bile acids regulate hepatic low density lipoprotein receptor activity in the hamster by altering cholesterol flux across the liver. Proc. Natl. Acad. Sci. USA. 83:1916-1920.

9. Shefer, S., S. Hauser, and E. H. Mosbach. 1968. 7alpha-hydroxylation of cholestanol by rat liver microsomes. J. Lipid Res. 9:328333.

10. Gustafsson, B. E., B. Angelin, K. Einarsson, and J. A. Gustafs- 
son. 1978. Influence of cholestyramine on synthesis of cholesterol and bile acids in germfree rats. J. Lipid Res. 19:972-977.

11. Eriksson, S. 1957. Biliary excretion of bile acids and cholesterol in bile fistula rats: bile acids and steroids. Proc. Soc. Exp. Biol. Med. 94:578-582.

12. Myant, N. B., and H. A. Eder. 1961. The effect of biliary drainage upon the synthesis of cholesterol in the liver. J. Lipid Res. 2:363-368.

13. Dowling, R. H., E. Mack, and D. M. Small. 1970. Effects of controlled interruption of the enterohepatic circulation of bile salts by biliary diversion and by ileal resection on bile salt secretion, synthesis, and pool size in the rhesus monkey. J. Clin. Invest. 49:232-242.

14. Redinger, R. M. 1984. The economy of the enterohepatic circulation of bile acids in the baboon. 2. Regulation of bile acid synthesis by enterohepatic circulation of bile acids. J. Lipid Res. 25:437-447.

15. Einarsson, K., K. Hellström, and M. Kallner. 1973. Feedback of bile acid formation in man. Metab. Clin. Exp. 22:1477-1483.

16. Einarsson, K., K. Hellström, and M. Kallner. 1974. Influence of deoxycholic acid feeding on the elimination of cholesterol in normolipaemic subjects. Clin. Sci. Mol. Med. 47:425-433.

17. Pomare, E. W., and T. S. Low-Beer. 1975. The selective inhibition of chenodeoxycholate synthesis by cholate metabolites in man. Clin. Sci. Mol. Med. 48:315-321.

18. Danzinger, R. G., A. F. Hofmann, J. L. Thistle, and L. J. Schoenfield. 1973. Effect of oral chenodeoxycholic acid on bile acid kinetics and biliary lipid composition in women with cholelithiasis. $J$. Clin. Invest. 52:2809-2821.

19. LaRusso, N. F., P. A. Szczepanik, and A. F. Hofmann. 1977. Effect of deoxycholic acid ingestion on bile acid metabolism and biliary lipid secretion in normal subjects. Gastroenterology. 72:132-140.

20. Nilsell, K., B. Angelin, B. Leijd, and K. Einarsson. 1983. Comparative effects of ursodeoxycholic acid and chenodeoxycholic acid on bile acid kinetics and biliary lipid secretion in humans. Gastroenterology. 85:1248-1256.

21. von Bergmann, K., M. Epple-Gutsfeld, and O. Leiss. 1984. Differences in the effects of chenodeoxycholic and ursodeoxycholic acid on biliary lipid secretion and bile acid synthesis in patients with gallstones. Gastroenterology. 87:136-143.

22. LaRusso, N. F., N. E. Hoffman, A. F. Hofmann, T. C. Northfield, and J. L. Thistle. 1975. Effect of primary bile acid ingestion on bile acid metabolism and biliary lipid secretion in gallstone patients. Gastroenterology. 69:1301-1314.

23. Bertolotti, M., N. Carulli, D. Menozzi, F. Zironi, A. Digrisolo, A. Pinetti, and M. G. Baldini. 1986. In vivo evaluation of cholesterol 7alpha-hydroxylation in humans: effect of disease and drug treatment. J. Lipid Res. 27:1278-1286.

24. Botham, K. M., M. E. Lawson, G. J. Beckett, I. W. Percy-Robb, and G. S. Boyd. 1981. The effect of portal blood bile salt concentrations on bile salt synthesis in rat liver. Biochim. Biophys. Acta. 666:238-245.

25. Davis, R. A., W. E. Highsmith, M. Malone-McNeal, J. Archambault-Schexnayder, and J. -C. W. Kuan. 1983. Bile acid synthesis by cultured hepatocytes: inhibition by mevinolin but not by bile acids. $J$. Biol. Chem. 258:4079-4082.

26. Kubaska, W. M., E. C. Gurley, P. B. Hylemon, P. S. Guzelian, and Z. R. Vlahcevic. 1985. Absence of negative feedback control of bile acid biosynthesis in cultured rat hepatocytes. J. Biol. Chem. 260:13459-13463.

27. Lee, M. J., D. V. Parke, and M. W. Whitehouse. 1965. Regulation of cholesterol catabolism by bile salts and glycyrrhetic acid in vivo. Proc. Soc. Exp. Biol. Med. 120:6-8.

28. Wilson, J. D., W. H. Bentley, and G. T. Crowley. 1969. Regulation of bile acid formation in intact animals. In Bile Salt Metabolism. L. Schiff, M. Carey, and J. Dietschy, editors. Thomas, Springfield, IL. 140-148.

29. Davis, R. A., C. A. Musso, M. Malone-McNeal, G. R. Lattier,
P. M. Hyde, J. Archambault-Schexnayder, and M. Straka. 1988. Examination of bile acid negative feedback regulation in rats. $J$. Lipid Res. 29:202-211.

30. Duane, C., A. P. McHale, and J. Neal Hamilton. 1988. Studies of feedback suppression of bile salt synthesis in the bile-fistula rat. $J$. Lipid Res. 29:212-214.

31. Heuman, D. M., C. R. Hernandez, P. B. Hylemon, W. M. Kubaska, C. Hartmann, and Z. R. Vlahcevic. 1988. Regulation of bile acid synthesis. I. Effects of conjugated ursodeoxycholate and cholate on bile acid synthesis in chronic bile fistula rat. Hepatology (Baltimore). 8:358-365.

32. Stange, E. F., J. Scheibner, C. Lutz, and H. Ditschuneit. 1988. Feedback regulation of bile acid synthesis in the rat by dietary versus intravenous cholate or taurocholate. Hepatology (Baltimore). 8:879886.

33. Singhal, A. K., J. Finver-Sadowsky, C. K. McSherry, and E. H. Mosbach. 1983. Effect of cholesterol and bile acids on the regulation of cholesterol metabolism in hamster. Biochim. Biophys. Acta. 752:214222.

34. Carulli, N., M. Ponz de Leon, F. Zironi, R. Jori, and P. Loria. 1980. Bile acid feeding and hepatic sterol metabolisms: effect of deoxycholic acid. Gastroenterology. 79:637-641.

35. Kinugasa, T., K. Uchida, M. Kadowaki, H. Takase, Y. Nomura, and Y. Saito. 1981. Effect of bile duct ligation on bile acid metabolism in rats. J. Lipid Res. 22:201-207.

36. Weis, E. E., and C. A. Barth. 1978. The extracorporal bile duct: a new model for determination of bile flow and bile composition in the intact rat. J. Lipid Res. 19:856-862.

37. Stone, B. G., S. K. Erickson, W. Y. Craig, and A. D. Cooper. 1985. Regulation of rat biliary cholesterol secretion by agents that alter intrahepatic cholesterol metabolism. Evidence for a distinct biliary precursor pool. J. Clin. Invest. 76:1773-1781.

38. Tietz, P. S., J. L. Thistle, L. J. Miller, and N. F. LaRusso. 1984. Development and validation of a method for measuring the glycine and taurine conjugates of bile acids in bile by high-performance liquid chromatography. J. Chromatogr. 336:249-257.

39. Kuipers, F., R. Havinga, H. Bosschieter, G. P. Toorop, F. R. Hindriks, and R. J. Vonk. 1985. Enterohepatic circulation in the rat. Gastroenterology. 88:403-411.

40. Vyvoda, O. S., R. Coleman, and G. Holdsworth. 1977. Effects of different bile salts upon the composition and morphology of a liver plasma membrane preparation: deoxycholate is more membrane damaging then cholate and its conjugates. Biochim. Biophys. Acta. 465:68-76.

41. Miyazaki, K., F. Nakayama, and A. Koga. 1984. Effect of chenodeoxycholic and ursodeoxycholic acids on isolated adult human hepatocytes. Dig. Dis. Sci. 29:1123-1130.

42. Cronholm, T., and J. Sjövall. 1967. Bile acids in portal blood of rats fed different diets and cholestyramine. Eur. J. Biochem. 2:375383.

43. Benacerraf, B., D. Bilbey, G. Biozzi, B. N. Halperin, and C. Stiffel. 1957. The measurement of liver blood flow in partially hepatectomized rats. J. Physiol. (Lond.). 186:287-293.

44. Hall, R., E. Kok, and N. B. Javitt. 1988. Bile acid synthesis: down-regulation by monohydroxy bile acids. FASEB (Fed. Am. Soc. Exp. Biol.) J. 2:152-156.

45. Kuroki, S., E. H. Mosbach, R. J. Stenger, B. I. Cohen, and C. K. McSherry. 1987. Comparative effects of deoxycholate and 7-methyldeoxycholate in the hamster. Hepatology (Baltimore). 7:229-234.

46. Ahlberg, J., B. Angelin, K. Einarsson, K. Hellström, and B. Leijd. 1977. Influence of deoxycholic acid on biliary lipids in man. Clin. Sci. Mol. Med. 53:249-256.

47. Low-Beer, T. S. 1979. Colonic bacteria, deoxycholate and biliary lipid. In Biological Effects of Bile Acids. G. Paumgartner, A. Stiehl, 
and W. Gerok, editors. MTP Environment Ltd., Lancaster, UK. 71-76.

48. Carulli, N., M. Ponz de Leon, P. Loria, R. Iori, A. Rosi, and M. Romani. 1981. Effect of the selective expansion of cholic acid pool on bile lipid composition: possible mechanism of bile acid induced biliary cholesterol desaturation. Gastroenterology. 81:539-546.

49. Marcus, S. N., and K. W. Heaton. 1986. Intestinal transit, deoxycholic acid and the cholesterol saturation of bile: three inter-related factors. Gut. 27:550-558.

50. Hofmann, A. F., S. M. Grundy, and J. M. Lachin. 1982. Pretreatment biliary lipid composition in white patients with radiolucent gallstones in the National Cooperative Gallstone Study. Gastroenterology. 83:738-752.
51. Salen, G., G. Nicolau, S. Shefer, and E. H. Mosbach. 1975. Hepatic cholesterol metabolism in patients with gallstones. Gastroenterology. 69:676-684.

52. Vlahcevic, Z. R., C. C. Bell, I. Buhac, J. T. Farrar, and L. Swell. 1970. Diminished bile acid pool size in patients with gallstones. Gastroenterology. 59:165-173.

53. Rutgeerts, P., Y. Ghoos, and G. Vantrappen. 1982. Kinetics of primary bile acids in patients with non-operated Crohn's disease. Eur. J. Clin. Invest. 12:135-143.

54. Rutgeerts, P., Y. Ghoos, G. Vantrappen, and J. Fevery. 1986. Biliary lipid composition in patients with non-operated Crohn's disease. Dig. Dis. Sci. 31:27-32. 